\title{
Virtual motion of real particles
}

\section{G. TR Y G G VASON}

Department of Mechanical Engineering, Worcester Polytechnic Institute, Worcester, MA 01609, USA

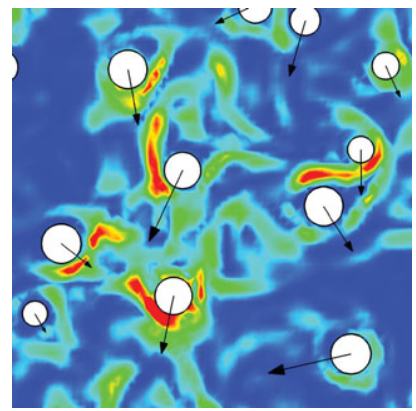

Direct numerical simulations are rapidly becoming one of the most important techniques to examine the dynamics of multiphase flows. Lucci, Ferrante \& Elghobashi (J. Fluid Mech., 2010, this issue, vol. 650, pp. 5-55) address several fundamental issues for spherical particles in isotropic turbulence. They show the importance of including the finite size of the particles and discuss how particles of a size comparable to the largest length scale at which viscosity substantially affects the turbulent eddies (i.e. the Taylor microscale) always increase the dissipation of turbulent kinetic energy.

\section{Introduction}

Multiphase flows play an important role in the workings of nature and the human enterprise. They are important, for example, in energy production, manufacturing, chemical processes, wastewater treatment and agriculture. Specific applications include riverbed erosions, rain, fluidized bed and bubble reactors, combustion of liquid fuels, heat transfer by boiling and many others. Such flows are notoriously hard to predict since analytical treatment is limited to only the simplest of cases and experiments are often difficult due to short length and time scales and limited optical access. It has been understood for a long time that computations hold the promise to revolutionize our understanding of multiphase flows. This is particularly true for direct numerical simulations (DNS), where all continuum length and time scales are fully resolved and the unsteady flow of a complex system with a range of length and time scales is simulated for long enough time so that it is possible to obtain a detailed statistical picture. In addition to yielding fundamental insight into the flow dynamics, such simulations provide information needed to develop model equations used to compute the average behaviour of industrial flows, where it is not possible to resolve the small-scale motion (Prosperetti \& Tryggvason 2007).

For single-phase turbulent flows the introduction of DNS was a paradigm shift of the first order that has resulted in information that earlier researchers could only dream of. For flows where DNS are possible, experiments have become unnecessary. Adding another phase increases the computational complexity in major ways. Although efforts to follow the unsteady motion of multiphase flows go back to the beginning of computational fluid dynamics (Harlow \& Welch 1965), new and improved methods developed in the late eighties and the early nineties provided the accuracy and robustness to make DNS of multiphase flow feasible. The key was the resurrection of an old idea: instead of attempting to modify the computational 
grid to align gridlines with a phase boundary, the grid remains fixed and stationary and the phase boundary is imbedded by replacing the singular interface with a smooth approximation (Harlow \& Welch 1965). The different phases are identified by a marker function that is either advected directly or constructed from connected marker particles that identify the interface and are advected with the flow. Early studies of the motion of rigid solids and deformable fluid particles can be found in Feng, Hu \& Joseph (1994) and Esmaeeli \& Tryggvason (1996). While confined to relatively small and simple systems, these studies allowed researchers to examine a much larger range of problems than previously possible. Earlier attempts to simulate multiphase flows had all focused on idealized systems, where the fluid inertia or the fluid viscosity was ignored and disperse flows of particles small enough so that they could be assumed to be point particles.

Although multiphase flows come in a wide variety of types, a large number of experiments and most modelling efforts have focused on disperse flows. This is partially because of their importance - it is difficult to suspend solids without breaking them into small pieces and in fluid-fluid systems one phase often breaks up into bubbles or drops - but also because they have long appeared to be more amenable to modelling. This continues to be the case and most DNS of multiphase flows have been concerned with suspended solid particles, drops and bubbles.

\section{Overview}

In the paper by Lucci, Ferrante \& Elghobashi (2010), the effect of particles on the decay of homogeneous turbulence is studied by DNS. The simulation is done on a fixed stationary grid, representing each particle using an immersed boundary marked by Lagrangian points distributed over the particle surface. The number of solid particles depends on their size and the mass loading and varies from 640 to 6400 . A closeup of the pressure distribution and the vorticity around one particle is shown in the left frame of figure 1. In the figure beside the title, two-dimensional contours of the spatial variation of the dissipation rate are shown along with the particles' velocity vectors in a subregion of the computational domain. It is clear that the size of the flow structures is comparable to the diameter of the particle.

Lucci et al. (2010) address several important issues. They note that point particle approximations are only justified under very restrictive conditions and fully resolving the flow around each particle is, in general, necessary. They have previously used the point particle approximation to study disperse multiphase flows with both solids (Elghobashi \& Truesdell 1992) and bubbles (Ferrante \& Elghobashi 2005) and thus are in an excellent position to comment on the importance of fully resolving the flow.

The effect of many solid or fluid particles on turbulence has been studied mostly in three scenarios: starting with initially quiescent flows, such as in the study of Bunner \& Tryggvason (2002) where the motion of over two hundred bubbles was simulated until the flow reached a statistically steady state; by maintaining the turbulence by adding a random force as done by a number of authors employing the point particle approximation (Cate et al. 2004); and by allowing the turbulence to decay naturally and examining the effect of the particles on the decay. Lucci et al. (2010) use the last approach, arguing that maintaining the turbulence by forcing artificially distorts the energy spectrum.

One of the major questions when small particles are suspended in turbulent flows is whether the addition of the particles increases or decreases the turbulent kinetic energy. Obviously large and heavy particles that fall through the fluid are likely to 

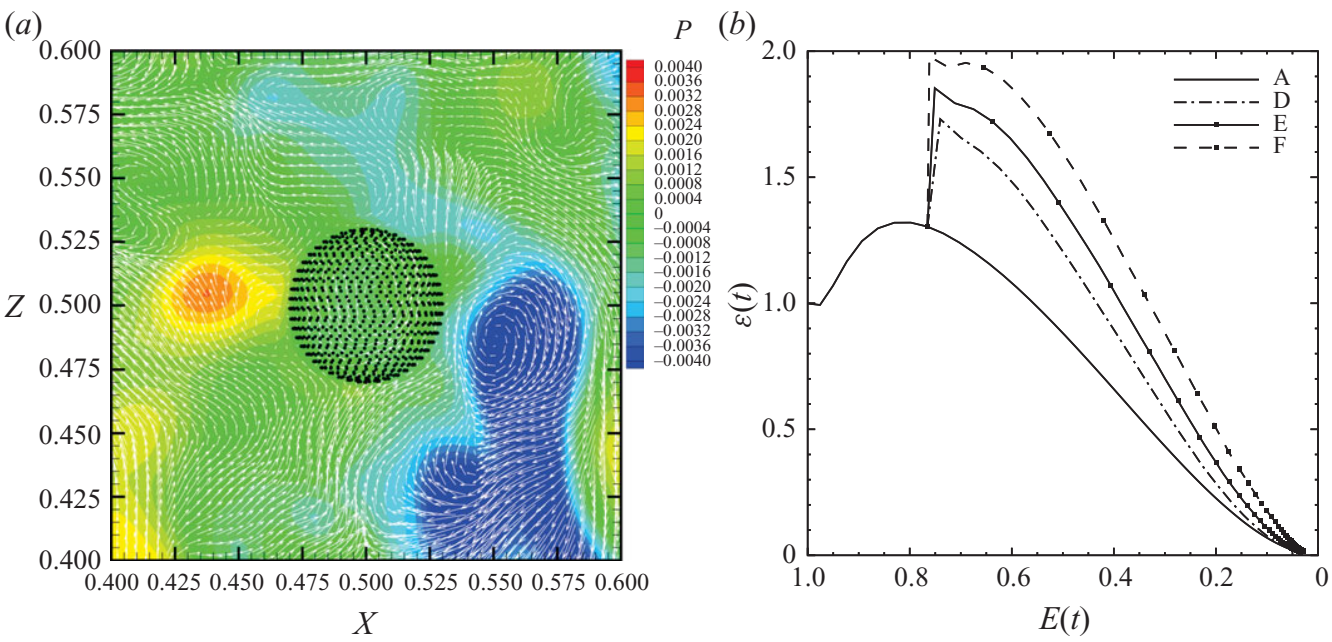

FiguRE 1. (a) Pressure distribution and velocity vectors around a spherical solid particle, represented by black dots on its surface, in isotropic turbulence. (b) Dissipation rate $\epsilon(t)$ plotted against turbulent kinetic energy $E(t)$ for the single-phase case (A) and three particle-laden cases with increasing mass fraction $(\mathrm{D}<\mathrm{E}<\mathrm{F})$.

generate turbulence. Small enough particles, on the other hand, increase the viscosity as Einstein showed - and thus are likely to increase the dissipation of the turbulence. Since the particle effects depend sensitively on the fluid flow in the immediate vicinity of the particle, the flow there must be resolved accurately. Lucci et al. (2010) do this and the results allow them to conclude that particles with a diameter of the order of the Taylor microscale (i.e. the eddy size where viscous effects start to have a non-trivial effect), always increase the dissipation of the turbulent kinetic energy. Figure 1(b) shows the interdependence of the instantaneous turbulent kinetic energy $E(t)$, and its dissipation rate $\epsilon(t)$, for the single-phase case (A) and three particle-laden cases with increasing mass fraction $(\mathrm{D}<\mathrm{E}<\mathrm{F})$. It is seen that $\epsilon(t)$ for the three particle-laden cases is always larger than that of case A for any value of $E(t)$.

The turbulent kinetic energy changes not only by viscous dissipation (which always leads to a decay) but also by the average rate of work done by the particles on the fluid (referred to as two-way coupling in the paper). Lucci et al. (2010) show that the rate of work is always a source of kinetic energy for the particle sizes that they consider, although the effect is always less than the viscous dissipation and does therefore not change the fact that the addition of the particles increases the rate of decay of the turbulent kinetic energy. For much smaller particles it is known that two-way coupling can be either positive or negative. Lucci et al. (2010) also examine the effect of other parameters such as the diameter of the particle and the density difference and then use the results to propose mechanisms to explain the results, an analysis only made possible by the availability from DNS of the complete flow field at every point in space and time.

\section{Future}

DNS are the biggest new development in multiphase flow research and while such simulations are already starting to have a major impact, they are still in their infancy. 
There are therefore abundant opportunities for such simulations to advance the field. For the relatively 'tame' problem of spherical particles in isotropic turbulence we do not understand what happens when the particles are larger, the mass loading is sufficiently high so that collisions are more frequent, if there is a distribution of particle sizes, or if the particles are not spherical. It seems likely, for example, that for large enough particles the production of kinetic energy due to two-way coupling will eventually be comparable to the increase in the viscous dissipation. As Lucci et al. (2010) demonstrate, we now have numerical methods that are capable of following the evolution of disperse multiphase flows and yield meaningful results for the statistical behaviour. The challenge is to make the most of these capabilities, gather results for systems that we can simulate and to advance the modelling of these systems.

Most multiphase flows of practical interest involve much more complex processes than spherical particles moving in incompressible Newtonian fluid. We want to know, for example, how a fuel jet breaks up into drops that then heat up, evaporate and burn; or, how boiling bubbles nucleate, grow, coalesce and carry vapour away from a hot surface. Robust and accurate numerical methods for flows with many competing physical processes (such as phase change, chemical reactions and so on) is currently taking place and within a few years we can expect to see DNS of flows that today can only be examined experimentally. The ability to examine complex flows with many competing processes in the detail currently possible for single-phase turbulent flows will be a real game-changer - to use a phrase currently in fashion. It will also require new ways to examine and condense the results and thus call for modelling in ways that we probably have not imagined yet.

\section{References}

Bunner, B. \& Tryggvason, G. 2002 Dynamics of homogeneous bubbly flows. Part 1. Rise velocity and microstructure of the bubbles. J. Fluid Mech. 466, 17-52.

Cate, A. T., Derksen, J. J., Portela, L. M. \& van den Akker, H. E. A. 2004 Fully resolved simulations of colliding monodisperse spheres in forced isotropic turbulence. J. Fluid Mech. 519, 233-271.

Elghobashi, S. \& Truesdell, G. C. 1992 Direct simulation of particle dispersion in a decaying isotropic turbulence. J. Fluid Mech. 242, 655-700.

Esmaeeli, A. \& TryggVason, G. 1996 An inverse energy cascade in two-dimensional, low Reynolds number bubbly flows. J. Fluid Mech. 314, 315-330.

Feng, J., Hu, H. H. \& JosePh, D. D. 1994 Direct simulation of initial value problems for the motion of solid bodies in a Newtonian fluid. Part 1. Sedimentation. J. Fluid Mech. 261, 95-134.

Ferrante, A. \& Elghobashi, S. E. 2005 Reynolds number effect on drag reduction in a bubble-laden spatially developing turbulent boundary layer. J. Fluid Mech. 543, 93-106.

HaRLow, F. H. \& WelCh, J. E. 1965 Numerical calculation of time-dependent viscous incompressible flow of fluid with a free surface. Phys. Fluid 8, 2182-2189.

Lucci, F., Ferrante, A. \& Elghobashi, S. 2010 Modulation of isotropic turbulence by particles of Taylor-length scale size. J. Fluid Mech. 650, 5-55.

Prosperetti, A. \& Tryggvason, G. 2007 Computational Methods for Multiphase Flow. Cambridge University Press. 